\title{
A NOTE ON EXTENSIONS OF FREE GROUPS BY TORSION GROUPS ${ }^{1}$
}

\author{
PAUL HILL
}

ABSTRACT. Under the assumption of the continuum hypothesis, a torsion free group $G$ is constructed that is the extension of a free group $F$ by a reduced primary group $T$ such that $G$ cannot be embedded in a product of $Z$ 's. In particular, $G$ is not free. This settles a question raised by Griffith.

In a paper just published, Griffith [3] has shown that if the torsion free abelian group $G$ is an extension of a free group $F$ by a torsion group $T$ then $G$ itself must be free provided that $T$ is weak $S_{p}$-projective for some reduced cotorsion functor $S_{p}$. In particular, this is the case if $T$ is a subgroup of a totally projective primary group. Specializing much further, one has that $G$ is free if $G$ is the extension of a free group $F$ by any countable, reduced primary group $T$. In [3] Griffith raised, and left unsettled, the following question: does the above result in fact hold for an arbitrary (uncountable) reduced, primary group $T$ ? In this note, we answer the question negatively. Unfortunately, our proof requires the continuum hypothesis, but we suspect that our result itself does not. At the very least we may save one from the futile effort of trying to generalize Griffith's theorem, without additional hypotheses, to an arbitrary reduced primary $T$.

Our notation is more or less standard; in particular, $c$ denotes the cardinality of the continuum and $\Omega$ denotes the first uncountable ordinal. If $B$ is a direct sum of cyclic $p$-groups, $\bar{B}$ denotes the torsion completion of $B$. In this connection, recall (from [2]) that any primary group $T$ without elements of infinite height is isomorphic to some pure subgroup between $B$ and $\bar{B}$, where $B$ is a basic subgroup of T. $\sum_{n<\omega} C\left(p^{n}\right)$ is called the standard basic $p$-group.

Theorem 1. Let $B$ be any unbounded basic p-group, that is, let $B$ be any unbounded primary group that can be written as a direct sum of cyclic groups. If the continuum hypothesis is assumed, then there exists a torsion free group $G$ that is not free but has a free subgroup $F$ such that $G / F=\bar{B}$, the torsion completion of $B$.

Received by the editors May 7, 1970.

AMS subject classifications. Primary 2030; Secondary 2048, 2051.

Key words and phrases. Torsion free abelian group, direct limits, free groups, extensions of groups, free groups by torsion groups.

1 This research was partially supported by NSF Grant GP-12318. 
Proof. Given $B$, we shall construct a group $G$ with the desired properties. By Lemma 50.1 in [2], it is enough to construct a nonfree group $G$ with a free subgroup $F$ such that $G / F$ is any primary group without elements of infinite height having $B$ as a basic subgroup. Furthermore, it suffices to do this for a countable $B$, for once we obtain such a group $G$ we can add to it an appropriate free group and get

$$
\bar{B}+\bar{C}=\overline{B+} \bar{C}
$$

as a homomorphic image, still with free kernel, where $C$ is arbitrary. Thus let $B$ denote an unbounded, countable $p$-group.

The key to the construction of $G$ is to do more than required; we shall actually construct $G$ such that it does not even have $Z$ as a direct summand. In particular, $G$ will not be separable, for it is necessarily $\boldsymbol{\aleph}_{1}$-free [3]. Before we begin the construction of $G$, let $G_{\alpha}$ denote, for each countable ordinal $\alpha$, the free group of countably infinite rank and let $B_{\alpha}$ denote an isomorphic copy of $B$. We shall obtain $G$ as a direct limit of the $G_{\alpha}$ 's. For simplicity of notation, we shall merely identify, in the appropriate manner, $G_{\alpha}$ in $G_{\beta}$ if $\alpha<\beta<\Omega$ and let $G=\bigcup_{\alpha<\Omega} G_{\alpha}$. Thus the construction of $G$ is accomplished essentially by the decision of how we shall identify $G_{\alpha}$ in $G_{\alpha+1}$ for each countable $\alpha$. Since we do not want $G$ to have a homomorphism onto $Z$, the choice of the identification of $G_{\alpha}$ in $G_{\alpha+1}$ will be heavily influenced by our desire to place obstruction to the extension to $G_{\alpha+1}$ of a map from $G_{\alpha}$ to $Z$. For each countable ordinal $\alpha$, denote by $E_{\alpha}$ the set of epimorphisms of $G_{\alpha}$ onto (a fixed) $Z$. There are exactly $c$ such epimorphisms. In view of the continuum hypothesis, the elements of $E_{\alpha}$ are in one-to-one correspondence with the countable ordinals; let

$$
E_{\alpha}=\left\{\pi_{\alpha, 0}, \pi_{\alpha, 1}, \cdots, \pi_{\alpha, \beta}, \cdots\right\}, \quad \beta<\Omega .
$$

Set $E=\bigcup_{\alpha<\Omega} E_{\alpha}$ and put $E$ into one-to-one correspondence with the countable ordinals with a function $f: E \mapsto[0, \Omega)$ such that $f\left(\pi_{\alpha, \beta}\right) \geqq \alpha$ for all $\alpha<\Omega$.

To begin the construction of $G$, let $\phi_{0}$ be an epimorphism of $G_{0}$ onto $B_{0}$ with kernel $K_{0}$,

$$
K_{0} \mapsto G_{0} \nrightarrow B_{0} .
$$

Let $\gamma<\Omega$. Suppose that we have an embedding of $G_{\alpha}$ into $G_{\beta}$, an embedding of $B_{\alpha}$ into $B_{\beta}$, and that we have an epimorphism $\phi_{\alpha}$ from $G_{\alpha}$ onto $B_{\alpha}$ if $\alpha \leqq \beta<\gamma$ such that the following conditions are satisfied:

(1) $G_{\beta} / G_{\alpha}$ is $p$-divisible if $\alpha<\beta<\gamma$. (2) $\phi_{\beta}$ is an extension of $\phi_{\alpha}$ and the kernel of $\phi_{\alpha}$ is a direct summand of the kernel of $\phi_{\beta}$ if $\alpha<\beta<\gamma$. 
(3) The map $\pi_{\alpha},{ }_{\beta}: G_{\alpha} \rightarrow Z$ cannot be extended to a homomorphism from $G_{\lambda}$ into $Z$ whenever $f\left(\pi_{\alpha, \beta}\right)+1=\lambda<\gamma$. (4) $B_{\alpha}$ is a basic subgroup (in the set-theoretic sense) of $B_{\beta}$ if $\alpha<\beta<\gamma$.

We wish to embed the $G_{\alpha}$ 's in to $G_{\gamma}$, the $B_{\alpha}$ 's into $B_{\gamma}$, and to produce an epimorphism $\phi_{\gamma}$ from $G_{\gamma}$ onto $B_{\gamma}$ in such a way that conditions (1)-(4) remain valid, respectively, for $\beta \leqq \gamma$ or $\lambda \leqq \gamma$. As usual, there are two cases depending on whether $\gamma$ is a limit ordinal or not.

Case 1. $\gamma$ is a limit. Set $B_{\gamma}=\bigcup_{\alpha<\gamma} B_{\alpha}$ and set $G_{\gamma}=\bigcup_{\alpha<\gamma} G_{\alpha}$; we remark that $B_{\gamma}$ and $G_{\gamma}$ are already structurally determined, but the union of the $B_{\alpha}$ 's is necessarily isomorphic to $B_{\gamma}$ in view of condition (4) and the union of the $G_{\alpha}$ 's is by Griffith's theorem [3] necessarily isomorphic to $G_{\gamma}$. Thus these identifications are admissible, and the induction obviously survives if we put $\phi_{\gamma}=\sup \left\{\phi_{\alpha}\right\}$. Note that the extension of (3) to $\lambda \leqq \gamma$ is vacuous because $\gamma$ is a limit ordinal.

Case 2. $\gamma-1$ exists. Let $\alpha$ and $\beta$ be the countable ordinals such that $f\left(\pi_{\alpha, \beta}\right)=\gamma-1$; recall that $f\left(\pi_{\alpha, \beta}\right) \geqq \alpha$, so $\alpha \leqq \gamma-1$. We know that $G_{\gamma-1}=\sum_{i<\omega}\left\{x_{i}\right\}$ is free and that $B_{\gamma-1}=\sum_{i<\omega}\left\{a_{i}\right\}$ is a direct sum of cyclic $p$-groups. According to [1], we can choose these decompositions such that $\phi_{\gamma-1}\left(x_{i}\right)=a_{i}$ for each $i$. As we already observed, there is no loss of generality in assuming that the order of $a_{i+1}$ is greater than the order of $a_{i}$ for each $i$-the same argument that was used in the reduction to a countable $B$ applies. Hereafter, we shall make this assumption. In what follows, if $\pi_{\alpha, \beta}$ cannot be extended to a homomorphism from $G_{\gamma-1}$ into $Z$, then its obstruction has already been obtained and the induction step simplifies. In particular, in that case, the nonnegative integer $N$ can be chosen arbitrarily. Note that if $\pi_{\alpha, \beta}$ does extend to $G_{\gamma-1}$ then its extension is unique because $G_{\gamma-1} / G_{\alpha}$ is $p$-divisible (and $Z$ is $p$-reduced). We shall continue to denote this unique extension by $\pi_{\alpha, \beta}$, so assume that $\pi_{\alpha, \beta}: G_{\gamma-1} \rightarrow Z$. Let $N$ be the smallest nonnegative integer such that $\pi_{\alpha, \beta}$ maps $\sum_{i \leq N}\left\{x_{i}\right\}$ onto $Z$. By the choice of $N$, we have $\pi_{\alpha, \beta}\left(x_{N}\right) \neq 0$. A crucial point is that we can assume that $\pi_{\alpha, \beta}\left(x_{i}\right)=0$ if $i>N$ and maintain the condition that $\phi_{\gamma-1}\left(x_{i}\right)=a_{i}$ for each $i$ because of the increasing orders of the $a_{i}$ 's. Denote the order of $a_{i}$ by $p^{e(i)}$ and set $e(i+1)=e(i)+d(i)$. Choose a prime $q \neq p$ such that $\pi_{\alpha, \beta}\left(x_{N}\right)$ has height zero in $Z$ at the prime $q$. Set $G_{\gamma}=\sum_{i<\omega}\left\{y_{i}\right\}$ and embed $G_{\gamma-1}$ into $G_{\gamma}$ according to the following relations:

$$
\begin{array}{ll}
x_{i}=y_{i} & \text { if } i<N, \\
x_{N}=q y_{N}+p^{d(N)} y_{N+1}, & \\
x_{i}=y_{i}+p^{d(i)} y_{i+1} & \text { if } i>N .
\end{array}
$$


Likewise, we set $B_{\gamma}=\sum_{i<\omega}\left\{b_{i}\right\}$ where $b_{i}$ has order $p^{e(i)}$, and we embed $B_{\gamma-1}$ into $B_{\gamma}$ by the relations:

$$
\begin{array}{rlrl}
a_{i} & =b_{i} & \text { if } i<N, \\
a_{N} & =q b_{N}+p^{d(N)} b_{N+1}, \\
a_{i} & =b_{i}+p^{d(i)} b_{i+1} \quad \text { if } i>N .
\end{array}
$$

It is well known and straightforward to verify that $\left({ }^{* *}\right)$ embeds $B_{\gamma-1}$ into $B_{\gamma}$ as a basic subgroup, so condition (4) holds for $\beta \leqq \gamma$. An immediate consequence of $\left({ }^{*}\right)$ is that $G_{\gamma} / G_{\gamma-1}$ is $p$-divisible. Thus condition (1) is satisfied for $\beta \leqq \gamma$. Clearly, the map $\phi_{\gamma}: y_{i} \rightarrow b_{i}$ extends $\phi_{\gamma-1}: x_{i} \rightarrow a_{i}$ because of the consistency of $\left({ }^{*}\right)$ and $\left({ }^{* *}\right)$. Furthermore, the kernel $K_{\gamma}$ of $\phi_{\gamma}$ is simply $\sum\left\{p^{e(i)} y_{i}\right\}$, whereas the kernel $K_{\gamma-1}$ of $\phi_{\gamma-1}$ is

$$
\sum_{i<N}\left\{p^{e(i)} y_{i}\right\}+\left\{q p^{e(N)} y_{N}+p^{e(N+1)} y_{N+1}\right\}+\sum_{i>N}\left\{p^{e(i)} y_{i}+p^{e(i+1)} y_{i+1}\right\} .
$$

Observe that $K_{\gamma}=\left\{p^{e(N)} y_{N}\right\}+K_{\gamma-1}$. Therefore, condition (2) is retained for $\beta \leqq \gamma$. In order to show that condition (3) remains valid for $\lambda \leqq \gamma$, we need to show only that $\pi_{\alpha, \beta}$ cannot be extended to a homomorphism of $G_{\gamma}$ into $Z$. Suppose that $\pi_{\alpha, \beta}$ extends to $G_{\gamma}$. Then $\pi_{\alpha, \beta}\left(y_{N+1}\right)=0$ since $x_{i}=y_{i}+p^{d(i)} y_{i+1}$ for all $i>N$ and since $\pi_{\alpha, \beta}\left(x_{i}\right)$ $=0$ for all $i>N$. Thus $\pi_{\alpha, \beta}\left(x_{N}\right)=q \pi_{\alpha, \beta}\left(y_{N}\right)$ which contradicts the fact that $\pi_{\alpha, \beta}\left(x_{N}\right)$ has zero $q$-height in $Z$. This completes the verification of the induction hypotheses.

Set $G=\bigcup_{\alpha<\Omega} G_{\alpha}, C=\bigcup_{\alpha<\Omega} B_{\alpha}$ and let $\phi=\sup \left\{\phi_{\alpha}\right\}_{\alpha<\Omega}$. Then $\phi$ is a homomorphism from $G$ onto $C$ and its kernel $K$ is free (because $K_{\alpha}$ splits out of $K_{\beta}$ if $\alpha<\beta$ and $K_{\alpha}$ is free for each $\alpha$ ). Obviously, condition (3) implies that $G$ admits no homomorphism onto $Z$. Condition (4) implies that $C$ has no elements of infinite height and that $B$ is a basic subgroup of $C$.

For the convenience of possible future reference, we record what we have proved as it relates to the standard basic $p$-group.

TheOREM 2. Let $B$ be the standard basic p-group. If the continuum hypothesis is assumed, then there exists a torsion free group $G$ that does not have $Z$ as a homomorphic image but has a free subgroup $F$ such that $G / F$ is between $B$ and $\bar{B}$.

COROLLARY. If the continuum hypothesis is assumed, there exists a (slender and $\boldsymbol{\aleph}_{1}-$ free) torsion free group $G$ that is the extension of a free group $F$ by a reduced primary group $T$ such that $G$ cannot be embedded in a product of $Z$ 's. 


\section{REFERENCES}

1. J. M. Cohen and H. Gluck, Stacked bases for modules, Bull. Amer. Math. Soc. 75 (1969), 978-979.

2. L.Fuchs, Abeliangroups, Akad. Kiad6, Budapest, 1958; republished by Internat. Series of Monographs on Pure and Appl. Math., Pergamon Press, New York, 1960. MR 21 \#5672; MR $22 \# 2644$.

3. P. Griffith, Extensions of free groups by torsion groups, Proc. Amer. Math. Soc. 24 (1970), 677-679.

Florida State University, Tallahassee, Florida 32306 